\title{
Les plateformes de la gratuité marchande et la controverse autour du Free Digital Labor : une nouvelle forme d'exploitation ?'
}

\author{
The "merchantable gratuitousness" platforms and the Free Digital Labor \\ controversy: a new form of exploitation?
}

\section{Carlo Vercellone ${ }^{1}$}

\author{
1 Université de Paris 8 Vincennes-Saint-Denis, CEMTI, Carlo.Vercellone@univ-paris8.fr
}

RÉSUMÉ. Le capitalisme cognitif et la révolution informationnelle sont allés de pair avec un effritement des frontières entre temps de travail et temps libre. Au centre de cette évolution se trouve l'essor du capitalisme des plateformes et notamment des plateformes de la " gratuité marchande " qui, à l'image de Google et Facebook, ont désormais conquis le premières places dans le classement des firmes mondiales en termes de capitalisation boursières et de rentabilité. Leur modèle de profit repose sur la logique des marchés multi-versants et associe la vente de la publicité en ligne et l'extraction des données des usagers. Ces derniers représentent ainsi à la fois le produit et les producteurs de la principale matière première à la base de l'organisation du marché publicitaire des plateformes de la gratuité marchande. C'est ce que l'on nomme le Free Digital Labor. Par ce concept on désigne le travail à la fois gratuit et apparemment libre qu'une multitude d'individus effectue sur internet, souvent inconsciemment, au profit des grands oligopoles du numérique et des data industries. La thèse du Free Digital Labor suscite une vive controverse. Elle est souvent rejetée au moyen de trois principaux arguments : ce serait, non le travail, mais le capital immatériel de l'algorithme qui, par un processus automatisé, extrairait et créerait l'essentiel de la valeur ; le Free Digital Labor échapperait non seulement aux critères canoniques du travail salarié, mais aussi à la définition anthropologique du travail vu comme une activité consciente et volontaire orientée vers un but ; les services gratuits offerts par les plateformes correspondraient à une rémunération en nature excluant tout rapport d'exploitation. Notre contribution se propose d'élucider les termes de ce débat et de répondre à ces objections par une analyse historique et théorique des mutations du rapport capital/travail intervenues sous l'égide du capitalisme des plateformes.

ABSTRACT. Cognitive capitalism and the informational revolution have gone hand in hand with a blurring of the boundaries between work and leisure time. At the heart of this evolution is the rise of platform capitalism, and in particular the "merchantable gratuitousness" platforms, like Google and Facebook, which have now taken first place in the ranking of world firms in terms of stock market capitalisation and profitability. Their profit model is based on the logic of multi-sided markets and combines the sale of online advertising and the extraction of user data. The users thus represent both the product and the producers of the main raw material underlying the organisation of the advertising market for merchantable gratuitousness platforms. This is called Free Digital Labor. This concept refers to the activity, apparently both gratuitous and self-governing, performed, often unknowingly, by a multitude of individuals on the internet for the benefit of big internet oligopolies and data industries. The Free Digital Labor thesis is highly controversial. It is often rejected by means of three main arguments: 1 . it would be, not labor, but the intangible capital of the algorithm which, through an automated process, would extract and create most of the value; 2. the Free Digital Labor would escape not only the canonical criteria of wage labor, but also the anthropological definition of labor as a conscious and voluntary goal-oriented activity; 3 . the free services proposed by the platforms would be remuneration in kind, excluding any relationship of exploitation. Our contribution aims to clarify the terms of this debate and to respond to these objections through a historical and theoretical analysis of the changes in the capital-labor relationship that occurred under the aegis of platform capitalism.

MOTS-CLÉS. capitalisme cognitif, capitalisme des plateformes, marches multi-versants, données, Algorithmes, Free Digital Labor, Karl Marx.

KEYWORDS. cognitive capitalism, platform capitalism, multi-sided markets, data, Algorithms, Free Digital Labour, Karl Marx

\footnotetext{
${ }^{1}$ L'auteur tient à remercier les deux rapporteurs de cet article pour leurs remarques et suggestions. Néanmoins, il reste seul responsable des éventuelles erreurs et imperfections restantes.
} 


\section{Introduction}

L'infrastructure de l'internet, conçue au départ comme un bien commun décentralisé et à vocation, pour l'essentiel, non marchande, fait aujourd'hui l'objet d'un puissant processus de recentralisation et de marchandisation [VER 18]. Avec une formidable accélération à partir de la crise de 2008, ce processus s'est notamment développé sous l'égide des plateformes des réseaux socio-numériques et des moteurs de recherche, à l'image de Google (Alphabet) et Facebook. Ces deux grands oligopoles de l'économie de l'internet et des data industries occupent désormais les premières places du capitalisme mondial en termes de capitalisation boursière et réalisent d'énormes bénéfices, tout en ne mobilisant qu'une masse quasi insignifiante de travailleurs salariés ${ }^{2}$.

Comment rendre compte de ce paradoxe inconcevable à l'âge du capitalisme industriel ?

L'explication de cette énigme a suscité durant cette dernière décennie des débats passionnés au centre desquels se trouve la question du digital labor, ou plus précisément de ce que l'on nomme le Free Digital Labor, c'est-à-dire l'activité pour l'essentiel, non rémunérée des usagers de plateformes des réseaux socio-numériques et des moteurs de recherche ${ }^{3}$.

Quelles sont les sources de la valeur et du pouvoir informationnel dont bénéficient des plateformes comme Google et Facebook?

Le Free Digital Labor peut-il être considéré comme un véritable travail tant au sens anthropologique de ce terme que des critères qui, depuis l'économie politique des classiques, définissent, dans le capitalisme, la notion de travail productif, celui d'une activité créatrice de marchandises et de profits?

Afin de répondre à ces questions et d'élucider les enjeux de ce débat, nous procèderons en deux étapes.

Dans la première, nous rappellerons les principaux traits qui différencient l'organisation du capitalisme industriel de celle du capitalisme de plateforme. Dans cette démarche, nous présenterons la thèse du Free Digital Labor en nous appuyant sur le modèle des plateformes de la "gratuité marchande » où le travail gratuit des usagers joue un rôle prépondérant dans la création de la valeur et des effets de réseau.

Dans la deuxième, nous nous pencherons sur la controverse concernant la nature et le rôle économique du Free Digital Labor. Par l'adoption d'une démarche que 1'on peut qualifier de «critique des critiques », nous examinerons les principales objections adressées au concept de Free Digital Labor à travers une analyse historique et théorique des mutations du rapport capital/travail intervenues sous l'égide du capitalisme des plateformes

\section{De l'entreprise fordiste au capitalisme des plateformes}

Le développement du capitalisme des plateformes incarne trois tendances majeures qui ont profondément déstabilisé la représentation traditionnelle de l'organisation de la firme et du travail à

\footnotetext{
${ }^{2}$ Au 31 décembre 2018, Alphabet et Facebook occupaient respectivement la quatrième et la sixième place dans le classement des principales firmes en termes de capitalisation boursière du Financial Times Global 500. Toujours en 2018 Facebook ne comptait qu'environ 30.000 salariés (https://newsroom.fb.com/company-info/) à travers le monde, Alphabet environ 85050.

${ }^{3}$ Le Free Digital Labor représente en quelque sorte la forme le plus pure du Digital Labor, celle d'une activité gratuite, immatérielle et pour l'essentiel en apparence libre et non contrainte. Il se déploie notamment dans les plateformes publicitaires de la gratuité marchande sur lesquelles nous focaliserons notre attention dans le cadre de cet article.
} 
l'âge du capitalisme industriel : l'effritement des frontières respectives entre temps de travail et temps de loisir ; hiérarchie et marché ; rente et profit ${ }^{4}$.

En effet, dans le modèle canonique du fordisme, la place centrale du capital matériel (machinesoutils, bâtiments etc.), et des matières premières elles aussi matérielles (charbons, pétrole, acier), allait de pair avec l'emploi d'une masse de force de travail salariée stable et concentrée dans les usines et les bureaux des corporations. Dans ce cadre, il existait également une distinction assez nette entre hiérarchie et marché. L'organisation de la firme produisait en interne les marchandises qui constituait le cœur de son métier en misant sur les rendements croissants autorisés par la division technique du travail et les économies d'échelle. Les marchandises étaient ensuite écoulées sur le marché auprès d'un public de consommateurs pour l'essentiel passif.

Ce modèle piloté par l'offre concernait les industries spécialisées dans la production de masse de biens d'équipements ménagers, mais aussi les industries culturelles (livres, disques, journaux, télévision) où la communication reposait sur un schéma unidirectionnel allant de l'émetteur ou producteur à une audience passive.

Les grands oligopoles de l'économie numérique et des datas industries sont parvenus à inventer un nouveau modèle de profit remettant en cause ces séparations entre organisation et marché, et sphère de la production et sphère de la consommation. Dans l'économie des plateformes prédomine ainsi un modèle quasiment inversé fondé sur trois piliers principaux.

1) Le principal capital fixe est de nature immatérielle ${ }^{5}$ et constitué, comme dans le cas de PageRank pour Google, et d'EdgeRank pour Facebook (au départ), d'un algorithme central et propriétaire, articulé à d'autres algorithmes. Dans l'économie des plateformes, c'est en effet la capacité de calcul et de traitement d'un torrent incessant d'informations (structurées et non structurées) qui détermine une partie essentielle de la force compétitive de la firme.

2) La principale matière première de l'économie des plateformes, elle aussi immatérielle, sont les big data, dont elles se servent dans différents buts qui peuvent se combiner. Par exemple, organiser leur activité en coordonnant l'action d'une multitude d'agents économiques ; faire directement de leur extraction et de leur élaboration le produit principal à vendre sous la forme d'espaces publicitaires ou sur le marché de plus en plus florissant des big-data; ou encore fournir des services aux autres entreprises grâce au know how acquis dans la maitrise des algorithmes et le traitement de données. C'est pourquoi les big data sont souvent considérés comme le nouveau pétrole de l'économie contemporaine et les plateformes comme les vecteurs d'une forme nouvelle d'extractivisme [MEZ 18].

3) Enfin, dans la plupart des firmes-plateformes le marché n'est pas une entité séparée de l'organisation. Mieux, elles peuvent être appréhendées comme une sorte d'hybride Entreprise-Marché

\footnotetext{
${ }^{4}$ Pour une analyse plus approfondie de la montée de la rente et du brouillage entre rente et profit dans le capitalisme contemporain cf. [ASK 16] [DUR 18], [VER 07] et [VER 19].

${ }^{5}$ Cette modification de la composition du capital est une tendance générale du capitalisme cognitif et informationnel : la part des actifs immatériels dans le calcul de la valeur de la capitalisation boursière des 500 principales firmes américaines serait passée, entre 1975 et 2005 , de $16,8 \%$ à $79,7 \%$. Ce ratio serait encore plus élevé, jusqu'à $90 \%$ pour les entreprises technologiques et notamment les GAFAM [CAS 08]. Néanmoins, la place centrale jouée par le capital nommé intangible (algorithmes, bases de données, brevets etc.) dans les grands oligopoles de l'internet et de data industries, ne doit pas nous faire oublier la manière dont leur filière productive articule en réalité étroitement, de l'amont à l'aval de sa chaîne de la valeur, matériel et immatériel, extraction de données et de ressources naturelles souvent non renouvelables [SMY 17]. En amont, car l'économie de l'immatériel et de l'internet est tributaire de la surexploitation de diverses matières premières, comme les terres et les métaux rares nécessaires pour les piles, les batteries et les écrans plats de nos ordinateurs et de nos smartphones ; en aval, car les besoins exorbitants créés par le profilage des usagers à des fins publicitaires ou de surveillance, conduisent à une surextraction et à une véritable suraccumulation de datas. Leur stockage, leur conservation et leur traitement repose sur la mise en place d'énormes infrastructures matérielles, à l'image des mégadatacenters des Gafam, dont le fonctionnement requiert une énorme consommation d'énergie avec un impact ravageur sur l'environnement.
} 
[CAS 19] brouillant les séparations traditionnelles que la théorie économique, depuis Coase [COA 37], avait établis entre hiérarchie et marché et l'alternative entre faire et faire faire, à savoir centraliser l'activité à l'intérieur de la firme ou la sous-traiter sur le marché. ${ }^{6}$ D'une part, grâce à leurs algorithmes, les plateformes internalisent en quelque sorte le marché à l'intérieur de l'entreprise, en en faisant une activité lucrative source de rentes d'intermédiation et de monopole. D'autre part, elles parviennent dans de nombreux cas à exercer des fonctions hiérarchiques de prescription et de contrôle du travail proches de celles de l'entreprise traditionnelle, même lorsqu'elles ont recours à un travail formellement indépendant (Uber, Deliveroo) ou pour l'essentiel gratuit, comme dans les réseaux socionumériques et les moteurs de recherche

Le rôle principal des plateformes consiste en fait à articuler les différents côtés interdépendants de ce qu'on appelle un marché biface [TIR 18], [ROC 03]) ou, plus précisément, multiversants [VER 18]. Celui-ci peut être composé soit par les acheteurs ou vendeurs d'un même bien ou service, soit par des acteurs aux buts très différents, comme c'est le cas, dans les plateformes publicitaires, pour les usagers et les annonceurs ${ }^{7}$. Dans tous les cas, la rentabilité de la firme dépend de sa capacité à connecter et à mobiliser le plus grand nombre d'usagers sur les différentes faces de la plateforme. Dans ce cadre, le rôle primordial joué jadis, dans le capitalisme industriel, par les économies d'échelle tend alors à être remplacé par les économies de réseau, comme l'indique l'association des lois de Metcalfe et de Reed selon laquelle la valeur d'un réseau est proportionnelle au carré du nombre de ses utilisateurs (loi de Metcalfe) et est démultipliée (loi de Reed) par la qualité et la densité de leurs interactions [REE 99], [OBE 03]. Nous avons là une condition déterminante de leur compétitivité et de la course vers la conquête d'une position de monopole et/ou de Monopsone.

Ces lois gouvernent à divers degrés tous les types de plateformes ${ }^{8}$. Toutefois c'est au premier chef dans les plateformes publicitaires, comme Google et Facebook, que la combinaison des logiques de la «gratuité marchande » et du Free Digital Labour est le véritable nerf de la guerre de cette stratégie qui vise à maximiser les externalités positives et la puissance productive des économies de réseau.

Pourquoi « gratuité marchande », un concept qui de prime abord peut apparaître comme un oxymore?

Car dans l'organisation des plateformes biface (ou multi-versants), cette gratuité n'est en réalité qu'instrument au service d'une logique propriétaire et lucrative. Il s'agit d'une relation économique «dans laquelle la gratuité n'a paradoxalement d'autre but que de permettre aux entreprises d'augmenter leurs profits » [FAR 15] p. 26. Autrement dit, des services en apparence gratuits sont offerts sur un versant de la plateforme aux internautes de manière à les attirer, à en extraire le maximum de données et de contenus, et cela afin de vendre sur l'autre versant des espaces publicitaires ciblés à des annonceurs qui s'adressent à ces mêmes usagers.

\footnotetext{
${ }^{6}$ Selon Ronald Coase [COA 37] l'origine de la firme trouverait son explication dans les coûts de transactions liés au marché, parmi lesquels les plus importants sont la rédaction des contrats et leur respect (quantité et qualités des prestations, prix, etc.). La firme par la hiérarchie, en internalisant la production et pouvant contrôler directement l'activité de ses salariés, limiterait drastiquement ces coûts de transaction et l'incertitude sur l'exécution des contrats. Ces gains doivent pourtant être mis en balance avec les coûts liés à la responsabilité de la coordination directe et à la surveillance des salariés dans le processus de production.

\begin{abstract}
${ }^{7}$ C'est pourquoi la notion de marché biface nous semble insuffisante pour caractériser la nature et la complexité du capitalisme de plateforme. En particulier, elle laisse supposer que le marché biface, avec son offre et sa demande, préexiste, en quelque sorte, à la firme, alors que celui-ci doit parfois être construit à travers un processus de production comme dans les cas de Google et Facebook. Nous lui préférons l'expression anglaise plus large de «Multi-sides plateforms » qui ne réduit pas nécessairement la firme-plateforme au rôle d'un simple intermédiaire, mais peut intégrer l'idée de l'organisation d'un véritable cycle productif. Nous reviendrons sur ce point dans la seconde partie de cet article en critiquant notamment certains aspects de l'approche de Jean Tirole.
\end{abstract}

${ }^{8}$ Pour une classification des divers types de plateformes (publicitaires, à la demande, d'e-commerce, en nuage, industrielles, de produit, etc.) cf. en particulier [SRN 18]. 
Le modèle de profit des plateformes de la "gratuité marchande », à l'instar de Google et Facebook, fait ainsi émerger dans sa forme la plus pure un trait central de l'économie politique de l'internet et des datas industries : la question du rôle du travail gratuit des usagers des plateformes du Web 2 et de ce que l'on nomme le Free Digital Labor. Cette forme nouvelle de travail a trait à la manière dont le Web 2 et l'essor du capitalisme des plateformes a permis aux oligopoles d'internet de dilater les frontières des firmes en y intégrant le marché, mais aussi et surtout la collaboration des usagers, ou, suivant une expression de plus en plus usitée, des prosumers. (ce terme étant justement la contraction du mot professional ou producer et du mot consumer).

Par le concept de Free Digital Labor (Terranova [TER 00], Pasquinelli [PAS 08], Fuchs [FUC 12], Scholz [SCH 12], Cardon et Casilli [CAR 15b], Casilli [CAS 16] \& [CAS 19], Simonet [SIM 18], Vercellone et alii [VER 18]) on désigne ainsi, en jouant sur le double sens du terme free, le travail à la fois gratuit et apparemment libre et autonome qu'une multitude d'individus effectue à travers et sur internet, souvent inconsciemment, au profit des grands oligopoles d'internet et des data industries. Ceux-ci seraient parvenus à créer des écosystèmes dans lesquels les usagers participent à la production de données, d'informations et de contenus valorisés ensuite par les firmes à travers la publicité ou la vente d'autres services. Dans ce modèle tout semble se passer comme si la firme-plateforme était parvenue à imposer aux usagers une sorte d'échange implicite et qui peut être résumé de la manière suivante en adaptant et en complexifiant l'ancienne formule employée pour l'audience télévisuelle: si c'est gratuit, c'est parce qu'en réalité vous êtes non seulement le produit, mais aussi les travailleurs qui, par une activité collective, en apparence libre et ludique, me permettent de vous fabriquer et de vous vendre comme des marchandises (en me fournissant les données, les contenus ainsi que, grâce aux économies de réseau, la taille du marché nécessaire pour attirer les annonceurs). Conclusion : dans la mesure où cette valeur n'est pas "redistribuée » aux internautes, on peut même considérer qu'il s'agit d'un travail exploité, et cela tant au sens de la théorie classique de la valeur-travail [FUC 14], que de la théorie néoclassique de la répartition, puisque la rémunération d'un travail gratuit est par définition inférieure à sa productivité marginale.

C'est le noyau dur de la thèse du Free Digital Labour en tant que nouvelle forme d'exploitation et ce qui la différencie, à notre sens, des approches de l'audience-marchandise initiées durant les années soixante-dix par Dallas W. Smythe [SMY 77] ${ }^{9}$.

\section{La controverse sur le concept de Free Digital Labor}

Cette thèse suscite de nombreuses controverses parmi les spécialistes de l'économie numérique [CNN 16], tant pour ce qui concerne ses fondements théoriques que pour ses implications en termes de justice sociale et de régulation de l'économie de l'internet. A l'idée selon laquelle le Free Digital

\footnotetext{
${ }^{9}$ La généalogie du concept de Free Digital Labour trouve ses racines dans l'économie politique de l'audience-commodity de Smythe [SMY 77]. Cet économiste canadien, bien avant la révolution informationnelle, avait voulu compléter l'analyse critique de l'école de la Frankfort (Adorno, Horkheimer) concernant les effets d'uniformisation de l'industrie culturelle, en soulignant un autre point central : les grands médias faisaient disparaître la frontière entre travailleur et consommateurs, car l'audience était vendue aux publicitaires. En ce sens, selon Smythe, la reproduction même de la force de travail devenait une source indirecte de la survaleur. Cette approche fait sans doute de Smythe un précurseur de l'économie de l'attention [CIT 14] et des théories du Free Digital Labor. Toutefois à l'époque où il écrit, la pertinence de cette thèse est un peu forcée, car le public demeurait dans une position pour l'essentiel passive et sans possibilités d'interaction. Dans le cas de la radio et de la télévision, si l'audience peut être pensée comme un produit, le public ne peut pas être considéré, à notre avis, comme un travailleur, à la différence de ce qui se passe aujourd'hui avec le Free Digital Labour. Preuve en est aussi le fait que dans la télévision classique le prix de la publicité était déterminé par une mesure essentiellement quantitative de l'audience qui demeurait peu connue dans sa composition qualitative et ses comportements. En ce sens, le modèle d'affaires des plateformes publicitaires de la gratuité marchande prolonge, mais modifie aussi profondément l'ancien modèle de l'audience télévisuelle, et ce pour deux raisons essentielles : les usagers sont des sujets actifs de la plateformes, des prosumers des données et de contenus, qui permettent d'aboutir à une publicité plus ciblée, prédictive et efficace que celle proposée par les médias traditionnels ; cela implique la mise en place d'un processus de production qui intègre étroitement l'activité des usagers en vue de l'élaboration de la marchandise finale ainsi que de sa réalisation sur le marché.
} 
Labor pourrait être considéré non seulement comme un travail, mais aussi comme un travail productif créateur de valeur, il est opposé trois principales séries d'objections. Nous allons à présent les présenter et discuter leurs arguments en mettant en exergue certaines de leurs limites et contradictions.

\subsection{Automatisation algorithmique de la production et/ou Free Digital Labor: Ie laboratoire secret de la création de la valeur dans le capitalisme des plateformes}

Une première série d'objections consiste à affirmer que c'est en réalité le capital immatériel de l'algorithme qui, par un processus automatisé, crée l'essentiel de la valeur en extrayant les données et en leur donnant un sens qui permet de les valoriser sur le marché de la publicité en ligne » [BRO 17], p. 8. Dans la même veine, et en forçant davantage le trait, Srnicek soutient que « nous devrions considérer les données comme la matière première à extraire et les activités des usagers comme sa source naturelle » [SRN 18], p. 45, à l'image de l'eau ou du pétrole qui préexistent dans les tréfonds de la terre. Les données ne seraient donc pas le produit du travail des usagers, mais celui des algorithmes. Autrement dit, précise-t-il, ma thèse « contrairement à celle de l'exploitation d'une main d'œuvre gratuite, consiste à dire que les plateformes publicitaires reposent sur l'appropriation de données comme matière premières. Une fois enregistrée et transformées en données, les activités des internautes se transforment en une matière première brute pouvant être raffinée et utilisée ensuite de multiples manières » (Ibidem, p. 61).

Les activités des usagers ne correspondraient alors qu'à des simples inputs informationnels alimentant des processus automatisés qui, eux seuls, créeraient une véritable matière première et une valeur économique exploitable par les plateformes.

Broca synthétise bien l'ensemble de ces arguments dans une formulation plus nuancée qui, sans nier tout rôle à l'activité des usagers, le relègue dans une position tout à fait marginale. "Le "travail" [guillemets de l'auteur] dont il est question [le digital labor $\mathrm{CV}$ ] ne consiste plus alors qu'à produire la « matière première » (les données) d'un processus, dont les opérations essentielles sont pratiquées par des algorithmes programmés par d'autres travailleurs. L'internaute fournit des données à Google en utilisant son moteur de recherche ; les algorithmes de la multinationale de Mountain View se chargent de les trier, de les organiser et de les valoriser sur le marché de la publicité en ligne ». [BRO 17] p. 8. Et pour appuyer sa thèse Broca fait appel à Dominique Cardon selon lequel c'est la transformation des données «par un mécanisme d'agrégation, de calcul, de comparaison, de filtre, de classement ou de recommandation qui leur confère un sens (pour les internautes) et une valeur (pour les plateformes) » [CAR 15a] p. 55. Finalement, poursuit Broca, « le rôle de l'internaute semble parfois être devenu celui d'un simple auxiliaire des algorithmes ; auxiliaire certes indispensable, mais dont la fonction est néanmoins relativement subalterne dans le processus de production de valeur » (Ibidem).

En synthèse, le Free Digital Labor, même si l'on admettait son existence, ne constituerait qu'une entité subalterne, presque insignifiante, d'émission de bits informationnels. Il se trouverait ainsi relégué dans une fonction de simple auxiliaire vis à vis du système automatique de la méga-machine algorithmique programmée par d'autres travailleurs.

Cette première série de critiques à la thèse du Free Digital Labor est affectée par deux erreurs majeures y compris si on l'analyse au prisme de l'approche des théories de la valeur travail dont aussi bien Broca que Srnicek semblent se réclamer.

La première erreur consiste à considérer le capital fixe immatériel incorporé dans les algorithmes, comme une source autonome de la création de valeur qui pourrait quasiment se passer de l'activité du digital labor des internautes. Or, le capital fixe ne peut créer en tant que tel de la valeur nouvelle, tant si l'on raisonne sur la base de la théorie classique de la valeur-travail que des conventions de la comptabilité nationale, selon laquelle la « valeur ajoutée nette » est égale au prix du produit, déduction faite des consommations intermédiaires et de l'amortissement du capital fixe, capital fixe dont les logiciels et les algorithmes font partie. En somme, les algorithmes, comme toute autre machine 
programmée pour exécuter un ensemble d'instructions, ne sont que du travail passé, cristallisé, peu importe si c'est dans un dispositif technique immatériel. De même qu'une machine-outil, ils ne constituent qu'une condition de la production qui facilite et encadre le travail humain et non pas un facteur autonome de création de valeur ${ }^{10}$. Ils ne seraient d'aucune utilité et demeureraient une ressource stérile sans le travail collectif des internautes qui l'alimente en matières premières et celui des programmeurs des algorithmes qui permet d'aboutir au produit fini. En somme, comme le rappelle Ciccarelli « L'activité qui alimente aujourd'hui le digital labor n'est pas accomplie exclusivement par une intelligence artificielle, mais par des légions d'hommes et de femmes face aux écrans de leurs ordinateurs allumés dans le monde entier [....]L'algorithme prospère grâce à la valeur produite par une force de travail » [CIC 18], p. 24.

Cette méprise est encore plus grande si l'on prend en compte deux autres observations complémentaires :

- Srnicek ne semble pas s'apercevoir qu'à la différence des ressources naturelles enfouies dans la terre, les inputs informationnels et les données brutes issues de l'activité des usagers ne sont pas un stock, mais un flux engendré en continu par l'activité des usagers. Il ne pourrait pas y avoir une démonstration plus flagrante de la façon dont elles sont le produit d'une activité incessante de travail, peu importe s'il est conscient ou inconscient de cet effet ${ }^{11}$. Srnicek semble par ailleurs le reconnaître lui-même - mais sans en tirer toutes les conséquences théoriques - lorsque dans un autre passage de son livre il affirme que «pour la plupart des plateformes, la qualité de données importe moins que leur quantité et diversité » tout en précisant que souvent «le moindre geste accompli par un utilisateurs contribue à reconfigurer les algorithmes et les processus d'optimisation » [SRN 18], p. 104, ce qui nous conduit à la seconde observation.

- en effet, une bonne partie de l'activité gratuite des internautes des médias sociaux ne se borne pas à produire des inputs informationnels enregistrés et élaborés par les algorithmes, mais consiste justement à entraîner ceux-ci à effectuer des tâches que l'intelligence artificielle n'est pas (ou pas encore) en mesure d'effectuer [CAS 19]. C'est à l'aune de cette interaction constante entre les usagers et le système de machines algorithmiques, ou comme le dirait Marx entre travail vivant et travail mort, que la nature de l'activité de travail des internautes doit être appréhendée.

La seconde erreur consiste à considérer que de toute manière la fonction de producteur de matières premières informationnelles ferait du digital labor une entité subalterne dans la chaîne de la création de la valeur des plateformes. Cette assertion ne peut que nous étonner à un âge où l'on commence à comparer les Big Data au nouveau pétrole ou au nouvel or du capitalisme cognitif et informationnel. Sur un plan historique elle frôle même l'absurde car elle reviendrait aussi à affirmer que les mineurs du charbon auraient eu une fonction secondaire à l'âge de la première révolution industrielle en Angleterre, ou encore que le pétrole n'aurait été qu'un élément marginal dans la civilisation de l'automobile.

Il en va de même lorsque pour amoindrir le rôle que le digital labor joue dans la création de valeur on le réduit à un simple auxiliaire de la machine algorithmique programmée par les ingénieurs en informatique et les data scientist des plateformes. On semble ainsi oublier que le travail taylorien de la chaîne de montage fordiste était, lui aussi, caractérisé par la plupart des sociologiques et économistes, comme un simple appendice des machines et de l'organisation scientifique du travail (OST) planifiée

\footnotetext{
${ }^{10}$ Sur le sens et l'importance de cette distinction théorique fondamentale chez Marx, comme chez Keynes, cf. [HAR 13] .

${ }^{11}$ Ce constat fait émerger une autre particularité de la matière première constituée par les data. Alors que les matières premières matérielles que l'on arrache à la terre sont des bien rivaux et des ressources limitées facilement excluables par les prix, le flux de données engendré par l'activité humaine est en principe une ressource infinie qui s'apparente à un bien collectif (non rival et difficilement excluable). Leur appropriation privée de la part des plateformes implique donc des mécanismes de cloturage les transformant artificiellement en ce qu'on appelle des biens de club ou à péage (non rivaux mais excluables par les prix).
} 
par les ingénieurs des bureaux de méthode. Personne n'aurait pour autant osé nier que c'était justement là, dans cette activité auxiliaire, mécanique et répétitive, que se trouvait le cœur du processus de création de valeur à l'âge fordiste.

\subsection{Une notion incompatible avec les fondements anthropologiques de la définition du travail ?}

Une seconde série d'objections adressées à la pertinence du concept de Free Digital Labor concernerait son incompatibilité avec le fondement anthropologique de ce que l'on nomme travail. Autrement dit, le concept de Free Digital Labor présenterait comme du travail «des activités que le sens commun ne considère pas comme telles» et il serait inconciliable « avec la définition philosophique moderne du travail en tant qu'activité consciente et volontaire ». [BRO 17], p. 1. Cette affirmation selon laquelle le Free digital labor ne serait pas un véritable travail car il échapperait au «sens commun» et ne serait pas dirigé consciemment par le travailleur vers un but choisi fait l'impasse sur trois points essentiels.

Tout d'abord, ce n'est évidemment pas la conscience subjective du concept de travail, encore moins le sens commun, qui le rends réel, mais plutôt l'acte même de travail qui s'inscrit dans des rapports sociaux et des institutions qui peuvent le rendre plus ou moins visible et reconnu ${ }^{12}$.

Il existe en effet une multitude d'exemples historiques d'activités remplissant pleinement les critères d'une définition anthropologique du travail et auxquelles ni le sens commun, ni la conscience de ses acteurs ne reconnaissent ce statut.

Ainsi à l'ère de la modernité, de la colonisation, et de la découverte du travail comme essence de l'homme (Hegel, Marx) et comme fondement de la propriété (Locke) nul doute que ce concept était étranger à la culture des populations des communs des chasseurs cueilleurs du « nouveau monde». Leur travail était tellement imbriqué aux autres activités sociales qu'il ne pouvait pas être isolé comme un acte particulier. Ces populations n'auraient même pas été en mesure de comprendre que leur activité de production et de reproduction de la communauté pouvait être qualifié de travail au sens occidental de ce terme. On se garda en effet bien de le leur dire pour pouvoir décréter que leurs terres communes correspondaient à une res nullius appropriable librement par les colons [WOO 09].

De même, pour prendre un autre exemple dont se revendiquent divers théoriciens du digital labor comme Scholz [SCH 12] et Simonet [SIM 18], une vaste littérature féministe a montré la manière dont le travail gratuit de reproduction réalisé par les femmes a été rendu durant longtemps, et souvent

\footnotetext{
${ }^{12}$ Dans la tradition de pensée initiée par Hegel, le travail, au sens anthropologique, est l'acte par lequel l'homme se crée lui-même au monde. En particulier dans les passages dédiés à la Dialectique du maître et de l'esclave, Hegel définit le travail comme la production de l'homme par lui-même. Par le travail l'esclave s'élève au-dessus de l'animalité et se montre supérieur au maître, en prouvant que l'homme n'a pas de nature fixe et immuable. Le travail devient ainsi l'instrument d'une libération possible qui affranchit l'esclave tout autant de la domination de la nature que de celle du maître. Prolongée par Marx, cette approche considère le travail comme l'essence même du genre humain. Les hommes, dans la reproduction des conditions de leur existence, coopèrent entre eux et agissent sur la nature, et en modifiant la nature transforment la nature même de l'homme. Plus précisément, comme il l'énonce à travers les célèbres métaphores de l'araignée et du tisserand et de l'abeille et de l'architecte, la dimension cognitive du travail, en tant qu'unité indissociable de la pensée et de l'action, est ce qui différencie l'homme de tous les autres êtres vivants dont l'activité est instinctuelle, mécanique et répétitive [MAR 93]. En ce sens, la norme capitaliste du travail salarié représente pour Marx la négation même du travail pensé comme libre expression de la créativité de l'homme et extériorisation de ses facultés physiques et intellectuelles. Rendu impersonnel et asservi à la logique du capital, le travail, de besoin humain essentiel, se mue en un simple moyen de gagner sa vie dans une activité subordonnée et aliéné dont le contenu, la nature et la finalité sont dictés par une puissance extérieure. Toutefois, selon Marx, la dimension anthropologique du travail, quoique niée par son aliénation sous le capital, continue à traverser tous les dualismes et les antagonismes qui structurent le rapport capital/travail et les catégories du capitalisme, de la marchandise au processus capitaliste de production : travail vivant et travail mort, travail concret et travail abstrait, procès de travail et procès de valorisation, comme nous le verrons dans la suite de notre analyse du concept de Free Digital Labor.
} 
aujourd'hui encore, invisible à la société et aux consciences mêmes de celles qui l'accomplissaient [FER 04] et [FER 11] .

Last but not least, aujourd'hui encore nombre d'activités créatrices de richesse et/ou de valeur ajoutée ne sont pas reconnues comme un véritable travail, ni par le sens commun, ni par la comptabilité nationale, pour le simple fait qu'elles échappent à la norme du rapport salarial et aux critères de mesure de l'emploi et du PIB. C'est par exemple le cas d'une multitude de stagiaires non rémunérés ou de bénévoles de l'économie du tiers secteur, tant et si bien que le sociologue Andrew Ross n'a pas hésité à intituler l'un de ses articles « le travail gratuit, stade ultime du capitalisme » [ROS 11].

L'absence de conscience du fait de réaliser un travail n'est pas en tout cas une particularité du digital labor. Ce problème d'identification et de reconnaissance du travail est d'autant plus grand que le capitalisme a conduit à créer progressivement une confusion majeure entre le concept de travail, au sens anthropologique de ce terme, et le concept de travail-emploi, i.e. un travail salarié subordonnée dont le mode d'exécution et les finalités sont hétéro-déterminées [GOR 88]. C'est pourquoi pour élucider la question de la nature comme de la perception subjective du travail, il est utile, à la suite de Marx [MAR 93], de rappeler comment le "processus de production" dans l'entreprise capitaliste présente une double face. Il est en fait l'unité contradictoire du procès de travail (travail concret), d'une part et du procès de valorisation (travail abstrait), d'autre part.

La première face, le procès de travail, désigne la manière dont les hommes, dans la reproduction des conditions de leur existence coopèrent et se servent de leur intelligence et de leurs outils (matériels et immatériels) pour satisfaire leurs besoins et exprimer leur subjectivité. C'est une condition universelle de l'activité humaine valable en tout type de société et qui correspond à la définition anthropologique du travail. Or à cet égard, celui du procès du travail, le Free Digital Labor se présente, dans la plupart des cas, comme une activité consciente et volontaire qui vise à produire des choses utiles et à exprimer la subjectivité des individus. Il en est sans doute le cas lorsque, par exemple, nous effectuons des actions aussi simples que celle d'effectuer une recherche sur Google pour trouver une information concernant la préparation d'un plat, l'histoire d'une ville, élaborer une bibliographie sur un sujet académique, donner un avis sur un livre ou encore poster un message pour organiser un évènement ou une réunion sur Facebook.

La seconde face, le procès de valorisation, désigne, elle, en revanche, la manière dont l'entreprise subordonne et réorganise le procès de travail pour le mettre au service de son objectif : la réalisation d'un profit au moyen de la production et de la vente de marchandises. Dans ce cadre, comme le précise Marx dans le chapitre six inédit du Capital le procès de travail n'est plus que le moyen du procès de valorisation, «celui-ci, comme tel, étant essentiellement production de plus-value, c'est-à-dire objectivation de travail non payé » [MAR 71], p.48. Or le point crucial est que ces deux faces du processus capitaliste de production peuvent se dissocier et ne pas apparaître simultanément à la conscience des acteurs. Nous pouvons illustrer cette dissociation cognitive par deux exemples extrêmes et opposés.

Le premier exemple est celui de la figure du travailleur salarié de la chaîne de montage, si bien incarnée par Charlie Chaplin dans les Temps Modernes. Celui-ci pouvait ne percevoir dans son activité que le seul côté inhérent au procès de valorisation, c'est-à-dire un travail mécanique, répétitif et asservi à un but extérieur qui vidait son travail concret de tout intérêt et le privait de toute vision d'ensemble du processus de production. Le travail n'apparaissait ainsi à l'ouvrier de la chaîne de montage qu'en tant que simple moyen de gagner sa vie et la perception de la dimension anthropologique du travail était quasiment effacée de sa conscience ${ }^{13}$.

\footnotetext{
${ }^{13}$ Il faut pourtant noter que même dans les formes les plus extrêmes de l'organisation scientifique du travail où la subjectivité du travailleur était tout simplement niée par le management, la dimension anthropologique du travail finissait par réapparaitre sous la forme de ce que les sociologues du travail ont qualifié d'implication paradoxale. En fait, comme le note Lipietz même l'ouvrier
} 
A un autre extrême, le second exemple est justement celui du prosumer d'internet qui, lui, à l'inverse, a souvent l'impression de n'accomplir qu'une activité pour soi, autodéterminée et relevant du temps libre. Il en résulte une conséquence paradoxale. D'une part, le prosumer ne considère pas son activité comme un véritable travail car elle échappe à la norme sociale dominante du travail-emploi subordonné et rémunéré, bien qu'elle participe à la création de la valeur appropriée par la plateforme. De l'autre, il la ressent cependant comme un acte dont il croît maîtriser l'objectif et le résultat, conformément au sens de la définition anthropologique du travail. En somme, de ce point de vue, ce qui fait défaut au digital labor c'est avant tout la conscience de la façon dont son travail s'inscrit aussi dans un procès de valorisation régis par une volonté extérieure et dirigé vers un but qui lui est dissimulé : celui de la création de marchandises et du profit.

Cette dissonance cognitive, cette aliénation, est d'autant plus forte que le mode de fonctionnement des plateformes capitalistes comme Google et Facebook est très éloigné de celui de la société disciplinaire et de la fabrique. Ce fonctionnement se rapproche plutôt de la description que Gilles Deleuze [DEL 90] faisait de l'émergence d'une société de contrôle, c'est-à-dire, un environnement technique invisible permettant de nous suivre partout et de nous orienter, sans jamais donner l'impression de véritablement nous contraindre ${ }^{14}$. On pourrait même affirmer que nous avons là une sorte de réalisation de l'utopie de tout manager ou dirigeant d'entreprise : disposer de travailleurs qui ont l'impression de ne travailler que pour eux-mêmes, et ce au point d'accepter de se mobiliser gratuitement au service de l'entreprise dans une sorte de servitude volontaire [GOR 97].

La perception de n'exécuter qu'une activité libre, exempte de tout rapport de domination et d'exploitation, est par ailleurs renforcée par la manière dont les prosumers semblent bénéficier gratuitement d'une panoplie d'outils et de services offerts par la plateforme et effectuer pour l'essentiel une activité ludique relevant du temps libre. Nous avons là le point de départ d'une autre critique adressée à la thèse du Free Digital Labor.

\subsection{La question de la contrepartie ou de la rémunération en nature}

Selon les managers ou les services de communication des plateformes mais aussi pour un certain nombre de chercheurs, la thèse du digital labor serait infirmée par l'existence d'une contrepartie en nature qui compenserait l'exploitation de la part des plateformes des données produites par les usagers. Ce constat ôterait donc au digital labor la nature d'un travail invisible non payé.

Jean Tirole, dans l'ouvrage où il se propose de livrer une synthèse de sa contribution à la Science Économique, résume de la manière suivant cet argument : «On entend souvent dire que les platesformes devraient payer pour les données que nous leur fournissons. En pratique cependant, certaines le font effectivement, non sous la forme d'un transfert financier mais sous la forme de services non tarifés. Nous échangeons de facto nos données contre la gratuité de services ancillaires (moteurs de recherche, réseaux socio-numériques, messagerie instantanée, vidéo en ligne) ou au cours de transactions commerciales (comme dans le cas d'Uber ou Airbnb). Les entreprises du web peuvent souvent arguer qu'elles ont dépensées de l'argent pour acquérir les données » [TIR 18], p. 546.

\footnotetext{
spécialisé (OS) « le plus taylorisé ne se contente pas d'obéir aux instructions du bureau des méthodes ou de suivre le mouvement de sa machine. Il utilise en permanence son imagination et son intelligence pour garantir la régularité du processus productif, en dépit des innombrables blocages causés par les produits semi-finis, les pannes, les disfonctionnements des machines, etc. Ce faisant, il exprime son autonomie en tant qu'être humain » [LIP 88]. Et sans cette «implication paradoxale », contradictoire et quasi inconsciente des OS, qui n'est pas sans présenter quelques analogies avec les comportements collaboratifs des prosumers des plateformes d'Internet, la chaîne de montage n'aurait pas pu fonctionner. Mais cette implication n'était pas reconnue dans l'organisation formelle de l'entreprise taylorienne ; elle ne l'est pas non plus aujourd'hui par le capitalisme des plateformes.
}

${ }^{14}$ Sur ce point cf. aussi l'analyse de l'univers des techniques de calcul des algorithmes proposée par Cardon [CAR 15a]. 
Cette objection semble en apparence imparable, mais à un examen plus attentif elle présente trois failles majeures.

Tirole, probablement influencé par le modèle des plateformes des cartes de débit ou crédit (Visa, American Express) dont l'analyse a joué un rôle essentiel pour sa contribution à la théorie des marches bi-face [ROC 02], définit l'ensemble des plateformes comme «ces entités qui permettent aux différents côtés (disons l'offre et la demande) du marché de se rencontrer et d'interagir », [TIR 18] (p. 508).

Les plateformes ne seraient en somme que des intermédiaires entre des acheteurs et de vendeurs d'un même produit ou service situés sur les deux faces du marché et qui, dans leur intérêt mutuel, bénéficieraient d'une baisse des coûts de transaction. Et selon Tirole, toutes les plateformes peuvent être intégrées dans ce modèle général de simple articulation entre des acheteurs et des vendeurs, comme le prix Nobel d'économie le résume dans une figure de l'ouvrage de synthèse de sa pensée [TIR 18] (p. 509) ${ }^{15}$. Si l'on suit ce schéma, de la même manière que les plateformes de système de paiement (carte de débit et de crédit) ne feraient que prélever une commission pour faciliter les transactions entre clients et commerçants, les plateformes des moteurs de recherche et des médias sociaux ne ferait que favoriser la rencontre entre des acheteurs (les utilisateurs) et des vendeurs (les annonceurs).

Il serait presque trivial de remarquer que cette analyse théorique est parfaitement conforme à l'image que les plateformes veulent donner d'elles-mêmes. Leur politique de communication repose sur un discours idéologique qui répète à l'envie qu'elles ne sont qu'un d'intermédiaire neutre entre les différentes faces du marché et les besoins de producteurs et des consommateurs qu'elles permettent de coordonner, ou encore de fournisseurs bienveillants de services technologiques gratuits pour l'épanouissement de la communauté mondiale des internautes ${ }^{16}$.

Mais l'essentiel n'est pas là. Plus fondamentalement, cette approche présente trois biais théoriques et empiriques majeurs :

a) Elle efface la grande diversité des modèles de profit et des sources de la valeur du capitalisme de plateforme. Ainsi si dans certains cas, comme dans le modèle des cartes de crédit ou à la limite de Airbnb, la fonction d'intermédiation apparaît effectivement comme l'élément essentiel, dans d'autres, comme Uber, Uber Eat et Deliveroo, cette thèse est beaucoup plus difficile à défendre. Dans ce cas, les plateformes ne se bornent pas à prélever une commission, mais fixent aussi les tarifs (et donc la rémunération) et exercent à l'égard des autoentrepreneurs des fonctions qui relèvent clairement de la hiérarchie de l'entreprise. De plus, Tirole semble oublier comment certaines d'entre elles ne sont pas des marchés bi-face, mais multi-versants, comme, par exemple Deliveroo ou Uber Eat où interagissent trois catégories d'acteurs : les livreurs autoentrepreneurs, les clients, mais aussi les restaurateurs.

\footnotetext{
15 Plus précisément, on fait référence à la Figure 1 à p. 159 intitulé Plates-formes bi-face [TIR 18], où chaque type de plateforme est présenté comme l'intermédiaire d'un couple Acheteurs-Vendeurs incarné dans les moteurs de recherche et les médias per le couple utilisateurs-annonceurs.

16 Ainsi, pour ne prendre qu'un exemple, Uber insiste avec force sur l'idée selon laquelle elle ne serait qu'un pur intermédiaire et non une entreprise de transport pour éloigner le risque d'une reconversion du statut de ses travailleurs autonomes en celui de travailleurs salariés. Facebook, elle, ne se présente pas comme une régie publicitaire, ni, par ailleurs, comme un intermédiaire entre deux faces d'un marché, mais comme le fournisseur d'un ensemble de services technologiques n'ayant pour but que de « vous connecter avec vos amis et votre famille, trouver des communautés et développer vos projets », comme l'affirme aussi dans le volet « qui sommesnous ? » de sa page de présentation en français. Le discours change bien évidemment et devient beaucoup plus réaliste lorsqu'on passe de la communication au public des usagers à la communication qui s'adresse aux actionnaires des grandes entreprises technologiques.
} 
b) l'activité des plateformes semble circonscrite à la seule sphère de la circulation, où elles joueraient le rôle d'une sorte de commissaire-priseur rémunéré, en excluant à priori tout rôle de la sphère de la production dans la création de la valeur ;

c) enfin, pour ce qui concerne plus particulièrement le sujet de cet article, Tirole ne semble pas s'apercevoir de la manière dont le modèle des plateformes de la gratuité marchande des moteurs de recherche et des réseaux socio-numériques est irréductible à un schéma acheteurs-vendeurs, et cela pour trois raisons étroitement imbriquées :

i) la motivation des usagers n'est sans doute pas celle d'acheter les marchandises proposées par les annonceurs ni d'être l'objet de leurs publicités, comme en témoigne par ailleurs le recours de plus en plus massif à des bloqueurs de publicité [CAR 19] qui impulsent, avec d'autres facteurs liés aux rendements décroissants de l'économie de l'attention, une baisse tendancielle de la rentabilité de la publicité en ligne [SMY 17].

ii) les plateformes publicitaires en se servant des traces, des données et des contenus engendrés par l'activité des usagers doivent donc transformer ces derniers en des cibles publicitaires plus attractives de l'audience classique proposée par la télévision et les autres médias traditionnels avec lesquelles elles entretiennent un rapport de concurrence sur le marché de la publicité. En somme, sur les plateformes de la gratuité marchande du type Google et Facebook, les usagers, loin d'être assimilables à des acheteurs, jouent en réalité un double rôle économique : celui de producteurs des matières premières et celui de la marchandise finale à vendre pour permettre à la plateforme de porter à terme la chaine de valeur issue de son cycle productif.

iii) la réalisation de cet objectif suppose bel et bien un processus de production qui en partant d'une série d'inputs (ressources humaines rémunérées et non rémunérées, machines algorithmiques et matérielles, matières premières, etc.) abouti à des outputs contenant une plus-value réalisable sur le marché. Dans ce cadre, les services ancillaires dont parle Tirole (moteurs de recherche, réseaux socio-numériques, messagerie instantanée, etc. etc.) doivent être spécifiquement conçus et programmés à cette fin en intégrant des algorithmes qui permettent de capturer les données des usagers et de les profiler comme des cibles publicitaires. Dans leur conception même, ces services ne sont donc en réalité pas des instruments techniques neutres, mais des moyens de productions mis en œuvre par les firmes-plateformes pour agencer le cycle productif en fonction de son procès de valorisation ${ }^{17}$.

\footnotetext{
${ }^{17}$ Askenazy semble aboutir à une conclusion semblable lorsqu'il affirme que dans les plateformes publicitaire « l'offre de multiples services n'a qu'un seul but : attirer le maximum d'internautes et leur soutirer le maximum d'informations sur eux-mêmes afin de bien cibler ces publicités » [ASK 16], p. 86-87.
} 


\section{Une représentation marxiste du cycle productif des plateformes de la gratuité marchande et du Free Digital Labor.}

Notons qu'au prisme de cette approche, les deux côtés des marchés bi-face de la gratuité marchande pourraient être facilement intégrés comme des étapes successives de la représentation que Marx donne du cycle du capital productif selon la séquence : A-M-P-M'-A', à savoir : du capital argent est investi dans des inputs productifs ( $\mathrm{M}$ comprenant les outils de production et la main-d'œuvre) pour le combiner dans un processus de production $(\mathrm{P})$ dont résulte une marchandise nouvelle (M') produite par l'entreprise qui incorpore une valeur supérieure à l'investissement initial en capital constant et variable ; enfin A' est la phase de la vente et de la réalisation de la plus-value sur le marché.

En adaptant cette formule du capital productif au modèle des plateformes des moteurs de recherche et des médias sociaux, nous aurions alors la séquence suivante : a) la face gratuite de la plateforme correspondrait à $\mathrm{A}-\mathrm{M}$, c'est à dire au capital constant et variable investi par la firme pour payer les programmeurs (et autres salariés), mettre en place ses moyens de production (tels les services ancillaires) et attirer les usagers ; b) P-M' correspondrait au processus de production permettant d'extraire de ses salariés et du travail gratuit des usagers la plus-value en transformant ces derniers en cibles publicitaires. C'est donc cette phase productive, et non la rencontre entre la demande des usagers et l'offre des annonceurs, qui relie les deux côtés de la plateforme. c) Enfin, A', correspondrait, elle, à la face payante de la plateforme où la plus-value est réalisée sur le marché de la publicité en ligne.

Encadré 1. Une représentation marxiste du cycle productif des plateformes de la gratuité marchande et du Free Digital Labor

En somme, si l'on raisonne du point de vue du procès de valorisation organisé par les plateformes de la "gratuité marchande", l'argument de la rémunération ou de la contrepartie en nature se présente sous une lumière bien différente. En réalité, l'infrastructure et les outils informationnels que les plateformes mettent à disposition des usagers jouent un rôle tout à fait comparable à celui des instruments de production que toute entreprise classique fournit à ses salariés afin qu'ils puissent exécuter sous sa direction les tâches pour lesquels ils sont employés. Or, personne ne songerait un instant à affirmer que dans une usine, par exemple, l'utilisation par les salariés des machines-outils ou d'autres instruments de production de propriété de l'entreprise pourrait constituer la juste contrepartie offerte gratuitement aux salariés pour leur travail ${ }^{18}$.

Cette remarque est d'autant plus vraie si l'on songe au fait qu'un account sur Facebook, sur Twitter et jadis sur Google+, ${ }^{19}$ n'est pas de propriété de l'usager. Il ne s'agit que d'un espace mis à sa disposition par la plateforme moyennant son consentement a lui céder les données qu'il produit, à améliorer les algorithmes et à se faire profiler sur la base de ses comportements, de ses traces, etc. L'usager peut risquer à tout moment de se faire éjecter du réseau et de se voir nier l'accès à sa page ou à son compte.

\footnotetext{
${ }^{18}$ C'est pourquoi Rey effectue, à notre sens, une erreur théorique lorsque pour défendre la thèse de l'exploitation du Free Digital Labor affirme que la valeur produite par les usagers est de toute manière supérieure à celle des services qui leur seraient offerts gratuitement [REY 12]. Il serait plus correct d'affirmer que la plus-value engendrée par les usagers permet d'obtenir un taux de profit positif compte tenu des investissements en capital constant et des coûts de production supportés par les entreprises.
}

${ }^{19}$ Jusqu'à sa fermeture définitive en avril 2019. 
Toutes les questions relatives à la propriété des moyens de production de la plateforme et des produits engendrés par l'activité des prosumers sont par ailleurs encadrées contractuellement par les conditions générales d'usage qu'il faut habituellement souscrire pour y avoir accès. Ainsi, dans une plateforme comme Facebook, ces conditions stipulent clairement que l'usager, doit céder à la plateforme la co-propriété des informations et des contenus qu'il a produit, sous la forme d'une licence transférable, sous-licenciable, gratuite, mondiale et «non exclusive» (mais qui de fait donne à Facebook un pouvoir quasiment illimité sur l'usage marchand ou non marchand de nos contenus et données). En somme, l'accès gratuit des usagers aux moyens de production de Facebook ou Google est subordonné à la reconnaissance du droit de l'entreprise à s'approprier du fruit de leur activité. Malgré l'absence d'une rémunération, nous avons là un trait commun essentiel qui rapproche le Free Digital Labor de la définition canonique du contrat salarial : l'aliénation de la part du travailleur de la propriété du produit de son travail ${ }^{20}$.

En somme, les activités menées par les internautes au moyen des services ancillaires des plateformes de la gratuité marchande sont en réalité assujetties à des rapports de propriété et à des normes contractuelles bien précises qui canalisent les comportements vers les objectifs de rentabilité de l'entreprise et rapprochent le Free Digital Labor d'une véritable forme de travail exploitée source de plusvalue.

\section{Conclusion}

Le capitalisme de plateforme n'est que la pointe la plus avancée d'un effritement général des frontières entre temps de travail et temps de loisir, production et consommation, sur lesquelles s'était construite la modernité capitaliste à l'issue de la révolution industrielle. Loin de correspondre à la réalisation de la énième prophétie de la fin du travail, l'automation algorithmique de la production accélère ce processus en nous menant vers une extension du travail qui empiète sur l'ensemble des temps sociaux et des interactions sociales des individus.

Dans cette évolution, le Free Digital Labor n'est pas en contradiction avec la définition philosophique moderne du travail au double sens que Marx attribue à ce concept : celui d'un travail concret, correspondant à son sens anthropologique ; celui d'un travail abstrait productif de plus-value.

Nous avons bien là, la solution de l'énigme dont nous sommes partis dans notre analyse, à savoir le paradoxe qui permet à des grandes entreprises technologiques comme Google et Facebook de se prévaloir d'une capitalisation boursière et de bénéfices énormes tout en demeurant des nains en termes d'emploi. C'est dans la mise au travail des prosumers de l'internet que se trouve « le laboratoire secret de la production» dont s'est nourri le développement du capitalisme des plateformes de la gratuité marchande.

Pour conclure, il importe aussi de préciser que, à l'instar de ce que Marx avait déjà remarqué pour le capitalisme industriel [MAR 93], la valeur dont s'approprient les propriétaires de Google et Facebook, n'est pas la simple somme de plusvalues individuelles, mais le produit de la coopération du travail, en l'occurrence celle de l'intelligence collective d'une multitude de prosumers. Ce n'est pas du simple like, post, video, recherche ou commentaire produit isolément par chaque usager, mais de leur interaction que surgit l'essentiel de la plusvalue. Ce que l'on nomme la « valeur du réseau », au sens de Metcalfe et de Reed, découle de cette force productive sociale du travail qui ne coûte rien au capital.

Toutefois, par rapport à l'âge de la fabrique et du capitalisme industriel, la coopération des prosumers de l'internet présente une différence majeure. Cette différence ne tient qu'à l'absence de

\footnotetext{
${ }^{20}$ Comme le fait remarquer Malo Morvan, il est très intéressant de noter que ce même type de conditions générales d'usage (même si nous ne le lisons souvent pas) concernent les chercheurs lorsqu'ils s'inscrivent et déposent des articles ou interagissent sur des plateformes « académiques » comme Academia edu [MOR 18]9.
} 
quasiment toute forme de rémunération, mais aussi et surtout à la nature de cette coopération. Dans les organisations du capitalisme industriel et de la société disciplinaire, la coopération du travail était planifiée par le capital qui contraignait les temps, les modes opératoires et l'enchaînement des différentes tâches et postes de travail. La technologie du capital se présentait, de manière inextricable, comme un instrument de production et comme un dispositif de disciplinarisation et de commandement direct sur le travail. Dans l'usine fordiste presque tout espace d'autonomie du procès de travail par rapport au procès de valorisation avait été effacé. C'est ce que Marx appelle la subsomption réelle du travail au capital [MAR 71]. Dans les organisations du capitalisme des plateformes et de la société du contrôle, la coopération doit être en revanche constamment incitée et canalisée, certes, mais aussi laissée libre de se déployer de façon autonome. C'est la conditio sine qua non pour maximiser l'efficacité des économies de réseau et extraire la masse et la qualité de données indispensable à leur procès de valorisation. Pour ce faire le capital fixe algorithmique de la firme-plateforme intercepte, trie, agrège et s'approprie des produits de l'activité des prosumers pour les transformer en marchandises et en valeur économique.

Nous sommes ici confrontés à un changement paradigmatique dans le rapport entre travail mort et travail vivant eu égard à l'âge du capitalisme industriel.

Dans les plateformes publicitaires, comme Google et Facebook, la technologie du capital s'apparente davantage à un «appareil de capture » des informations et de la valeur produites par l'activité des usagers, à une enveloppe dont les dispositifs sont sur bien des aspects superfétatoires par rapport aux conditions socio-techniques qui leur permettraient autrement de fonctionner tout simplement comme un moteur de recherche et un média socio-numérique, sans extraction de données, exploitation, violation de la privacy et surveillance. En synthèse, le procès de valorisation se greffe sur le procès de travail, mais ces deux faces ne se confondent plus dans un tout unique entièrement forgé par le capital «à son image et à sa ressemblance».

Cela contribue à expliquer, comme on l'a vu, la dissociation cognitive dont est victime une majorité des prosumers des plateformes de la gratuité marchande lorsqu'ils pensent n'exercer qu'une activité libre sans s'apercevoir qu'ils participent en même temps au procès de valorisation de l'entreprise.

Au sens de Marx, comme aux débuts du capital, la subsomption du travail au capital prend un caractère formel et peut sembler presque accidentelle. Le capitalisme vampire caractéristique du capitalisme industriel ne disparaît pas, loin de là. Il s'imbrique de plus en plus à un capitalisme parasite où la captation de la plusvalue s'opère à partir d'une coopération du travail potentiellement autonome et qui pourrait s'auto-organiser en dehors du capital.

C'est la raison pour laquelle la production de données et de valeur de la part des usagers des plateformes peut apparaître comme l'effet indirect d'une activité accomplie avec une autre intention.

C'est aussi la raison pour laquelle l'économie politique de l'internet demeure traversée par des pratiques contradictoires et des logiques de développement alternatives qui, à l'image du mouvement du coopérativisme de plateforme et du free-software, pourraient parvenir à déstabiliser l'hégémonie du capitalisme de plateforme en renouant avec l'esprit du commun de l'internet des pionniers ${ }^{21}$.

\section{Bibliographie}

[ASK 16] Askenazy PH., Tous rentiers! Pour une autre répartition des richesses, Odile Jacob, Paris, 2016.

[BRO 17] BROCA S., «Le digital labour, extension infinie ou fin du travail ? », Tracés, Revue de Sciences Humaines, p. 133-144, 2017.

[CAR 15a] CARDON D., A quoi rêvent les algorithmes, Seuil, Paris, 2015.

${ }^{21}$ Pour un panorama de principaux modèles alternatifs et une analyse de leur potentiel de développement cf. [VER 18]. 
[CAR 15b] CARDOn D., CASILli A., Qu'est-ce que le Digital Labor ?, INA Éditions, Bry-sur-Marne, 2015.

[CAR 19] CARDON D., Culture numérique, Sciencespo-Les Presses, Paris, 2019.

[CAS 08] CENTRE D'ANALYSE STRATÉGIQUE, «Évaluation et valorisation financière de la propriété intellectuelle : nouveaux enjeux, nouveaux mécanismes », La note de veille, $\mathrm{N}^{\circ} 111$, Octobre 2008. En ligne : http://archives.strategie.gouv.fr/cas/system/files/noteveille111.pdf (consulté le 5 avril 2020)

[CAS 16] CASILli A., "Le digital labor: une question de société », Rédaction INAGLOBAL, 2019. En ligne: http://gesd.free.fr/casilli116.pdf (consulté le 29 mars 2020).

[CAS 19] CASILLI A., En attendant les robots, Seuil, Paris, 2019.

[CIC 18] CiCCARELli R., Forza Lavoro. Il lato oscuro della rivoluzione digitale, Derive/Approdi, Roma, 2018.

[CIT 14] CitTon Y., L'économie de l'attention: Nouvel horizon du capitalisme ?, La Découverte, Paris, 2014.

[CNN 16] CONSEIL NATIONAL DU NUMÉRIQUE, Travail emploi numérique : les nouvelles trajectoires, Rapport rendu au Ministre du Travail, de l'Emploi, de la Formation professionnelle et du dialogue social, 6 janvier, 2016.

[COA 37] COASE R., « The nature of the firm », Economica, № 4, p. 386-405, 1937.

[DEL 90] Deleuze G., « Post-scriptum sur les sociétés de contrôle », dans Pourparlers, Les éditions de Minuit, Paris, 1990. En ligne : https://sites.google.com/site/deleuzemedia/textes/post-scriptum-sur-les-societes-de-contrôle (consulté le 29 mars 2020).

[DUR 18] DURAND C., "L'envers de l'économie numérique : Un capitalisme intellectuel monopoliste », Note de recherche IFRIS $\mathrm{N}^{\circ}$ 7, 2018. En ligne : http://ifris.org/wp-content/blogs.dir/1/files/2018/05/WP7-Cédric-Durand.pdf (consulté le 7 avril 2020).

[FAR 15] FARChy J., MÉAdel C., SiRE G., La Gratuité, à quel prix ? Circulation et échange de biens culturels sur Internet, Presses des Mines, Cahiers de l'EMNS, Paris, 2015.

[FED 11] FEDERICI S., « Women, Land Struggles, and the Reconstruction of the Commons », Working USA, The Journal of Labor and Society (WUSA), vol. 14, 1, p. 41-56, 2011.

[FER 04] FEDERICI S., Caliban and the Witch: Women, the Body and Primitive Accumulation, Autonomedia, Brooklyn, NY, 2004.

[FUC 12] FUCHS C., «Dallas Smythe today: The audience commodity, the digital labour debate, Marxist political economy and critical theory », TripleC, 10(2), p. 692-740, 2012. En ligne: http://www.triplec.at/index.php/tripleC/article/view/443 (consulté le 29 mars 2020).

[FUC 14] FUCHS C., Digital Labour and Karl Marx, Routledge, New York, 2014.

[GOR 88] GORZ A., Métamorphoses du travail, Quête du sens - Critique de la raison économique, éd. Galilée, Paris, 1988.

[GOR 97] GORZ A., Misère du présent, richesse du possible, éd. Galilée, Paris, 1997.

[HAR 13] HARRIBEY J-M., La richesse, la valeur et l'inestimable. Fondements d'une critique socio-écologique de l'économie capitaliste, Les Liens qui Libèrent, Paris, 2013.

[LIP 88] LIPIETZ A., «L'Après-Fordisme et son espace», Les Temps Modernes, N. 5, Avril, p. 75-114, 1988. http://lipietz.net/IMG/article_PDF/article_381.pdf, (consulté le 29 mars 2020)

[MAR 71] MARX K., Un chapitre inédit du capital, Traduction de l'Allemand et présentation de Roger Dangeville, Union générale d'Éditions, Collection Le Monde, Paris, 1971. En ligne : http://classiques.uqac.ca/classiques/Marx karl/capital chapitre inedit/capital chapitre VI inedit.pdf (consulté le 29 mars 2020).

[MAR 93] MARX K., Le Capital, Livre I, Quadrige, PUF, Paris, 1993.

[MEZ 18] MEZZADRA S., NEILSON B, «Entre extraction et exploitation : des mutations en cours dans l'organisation de la coopération sociale », Actuel Marx, n. 63, p. 97-113, 2018.

[MOR 18] MORVAN M., «Pourquoi je ne suis (presque) pas sur Academia.edu», 2018. En ligne : http://malomorvan.free.fr/textes/academia.pdf (consulté le 29 mars 2020).

[OBE 03] OBERSON P., La genèse des communautés virtuelles, Mémoire de Diplôme d'Études Supérieures Spécialisées, Université de Genève, 2003. En ligne : http://tecfa.unige.ch/tecfa/maltt/memoire/Oberson2003.pdf (consulté le 29 mars 2020). 
[PAS 08] PASQuinelli M., Animal Spirits: A Bestiary of the Commons, NAi Publishers / Institute of Network Cultures, Rotterdam, 2008.

[REE 99] ReED D. P., «That Sneaky Exponential: Beyond Metcalfe's Law to the Power of Community Building », Context Magazine, spring, 1999. Http://www.contextmag.com/archives/199903/digitalstrategy reedslaw.asp, consulté le 29 mars 2020).

[REY 12] REY P.J., « Alienation, Exploitation, and Social Media », American Behavioral Scientist, vol. 54, n 4, p. 399420, 2012.

[ROC 02] Rochet J.C., TIROLE J., « Cooperation among Competitors: Some Economics of Payment Card Associations », Rand Journal of Economics, 33(4), p 549-570, 2002.

[ROC 03] RocheT J.C., TIROLE J., « Platform Competition in Two-Sided Markets », Journal of the European Economic Association, vol. 1, no 4, p. 990-1029, 2003.

[ROS 11] Ross A., "Le travail gratuit, stade ultime du capitalisme », Courrier international, 2011. En ligne: https://www.courrierinternational.com/article/2011/06/09/le-travail-gratuit-stade-ultime-du-capitalisme (consulté le 29 mars 2020).

[SCH 12] SchOLZ T., Digital Labour: The Internet as Playground and Factory, Routledge, New York, 2012.

[SIM 18] SIMONET M., Travail gratuit : la nouvelle exploitation, Textuel, Paris, 2018.

[SMY 17] SMYRNAIOS N., Les GAFAM contre l'internet. Une économie politique du numérique, INA, Paris, 2017.

[SMY 77] SMYTHE D.W., «Communications: Blindspot of Western Marxism », Canadian Journal of Political and Society Theory, 1 (3), p. 1-28, 1977.

[SRN 18] SRNICEK N., Capitalisme de plateforme, Lux, Montréal, 2018.

[TER 00] TeRranova T., «Free Labor. Producing Culture for the Digital Economy », Social Text, Vol. 18, n. 2, p. 33$58,2000$.

[TIR 18] TIROLE J., Économie du bien commun, PUF, Paris, 2018.

[VER 07] VERCELLONE C., «La nouvelle articulation rente, salaire et profit dans le capitalisme cognitif », European Journal of Economic and Social Systems, Vol. 20, n¹, p. 45-64, 2007.

[Ver 18] Vercellone C. (dir), Brancaccio F., Giuliani A., Puletti F., Rocchi G., Vattimo P., Data-driven distruptive commons-based models, Report D 2.4, DECODE (Decentralised Citizens Owned Data Ecosystem), programme européen Horizon 2020, axe Information and Communication Technologies, October 31st, 2018. En ligne: https://decodeproject.eu/publications/data-driven-disruptive-commons-based-models-0 (consulté le 29 mars 2020).

[VER 19] VerCELlone C., Dughera S., « Metamorphosis of the theory of value and becoming-rent of profit: An attempt to clarify the terms of a debate », dans Cognitive Capitalism, Labour and Welfare. The Commonfare Hypothesis, Routledge, London, pp. 33-60, 2019.

[WOO 09] WoOD E.M., L'origine du capitalisme, Une étude approfondie, Lux, Québec, 2009. 